Ann. Biol. anim. Bioch. Biophys., 1977, 17 (5 B), 787-798.

\title{
Étude morphologique, chez le rat, du tissu adipeux périrénal au stade de sa formation
}

\author{
par Françoise DESNOYERS \\ avec la collaboration technique de S. DELPAL et G. PAILLARD
}

Station de Recherches de Nutrition, I.N.R.A., 78350 Jouy-en-Josas.

Summary. Morphological study of rat perirenal adipose fissue in the formative stage.

Rat perirenal adipose tissue is histocytologically studied since the moment of its appearance.

In this species, the first cells with lipid inclusions appear at about parturition time. They are always located in well-defined sites of the kidney region which are the hilus, the convex part of the perirenal facia, behind and below both kidneys and between them.

In the rat, the structural aspect of adipose tissues at different sites is the same, but electron microscopy reveals that cells have a different ultrastructure from the time they appear, depending on where they are located in the kidney region. Those situated between the kidneys (a localization of brown adipose tissue in this species) present a particularly different ultrastructural aspect from the time they appear.

We observe also that as the perirenal adipose tissue makes its initial appearance, the preadipocytes are numerous and aggregated around capillaries before they rapidly become filled with lipids.

Finally, we confirm that the cells of the white perirenal adipose tissue, like those of other white adipose tissues studied previously, are of endothelial origin.

\section{Introduction.}

Les investigations morphologiques concernant les tissus adipeux internes à graisse blanche ont été relativement nombreuses au cours des quinze dernières années, tant du point de vue structural (Simon, 1962 ; Wassermann, 1965 ; Lemonnier, 1972 ; Hermans, 1973) que du point de vue ultrastructural (Sheldon, Hollenberg et Winegrad, 1962 ; Napolitano, 1963 ; Napolitano, 1965 ; Sheldon, 1965 ; Sheldon, 1969 ; Vodovar, Desnoyers ef François, 1971 ; Vodovar, Desnoyers et Flanzy, 1972 ; Desnoyers et Vodovar, 1973 ; Desnoyers et Vodovar, 1974 ; Desnoyers, Vodovar ef Durand, 1975).

Les renseignements obtenus, essentiellement par l'étude cytologique des cellules adipeuses (Napolitano, 1963 ; Wassermann et McDonald, 1963 ; Vodovar, Serres ef François, $1969 \mathrm{~b}$; Vodovar, Desnoyers el François, 1971) ont fait progresser la con- 
naissance des tissus adipeux éludiés. Pour une même espèce, des différences sont observées selon l'emplacement du tissu adipeux dans l'organisme, suivant l'état nutritionnel de l'animal et suivant son âge (Lemonnier, 1972 ; Desnoyers, 1973 ; Desnoyers et Vodovar, 1974 ; Nouguès, 1975). Cette dernière constatation est très importante car elle permet de mettre fin à l'assimilation des tissus adipeux les uns pour les autres.

Depuis plusieurs années, nous avons entrepris l'étude systématique des tissus adipeux des différents emplacements de l'organisme chez le porc et chez le rat aux divers stades de la croissance (Vodovar, Serres et François, 1969a ; Vodovar, Serres ef François, 1969b; Vodovar, Desnoyers ef François, 1971 ; Vodovar, Desnoyers et Flanzy, 1972 ; Desnoyers, 1973 ; Desnoyers et Vodovar, 1973 ; Desnoyers, Vodovar et Durand, 1973 ; Desnoyers ef Vodovar, 1974 ; Desnoyers, Vodovar et Durand, 1975). Nous exposerons, ici, les observations effectuées chez le rat sur le tissu adipeux périrénal au stade de son apparition et de son premier développement et nous comparerons les résultats à ceux oblenus lors d'une étude préalable du tissu adipeux périrénal de porc au même stade de développement.

\section{Matériel et méthodes.}

Des observations préliminaires nous ont montré que dans la région rénale, la lipogenèse apparaît aux environs immédiats de la naissance chez le rat.

Les fœtus de rat ont été obtenus par césarienne 24 h et 12 h avant le jour présumé de la naissance. Par la suite, des ratons nouveau-nés, puis des ratons âgés de $12 \mathrm{~h}$, $24 \mathrm{~h}$ et $48 \mathrm{~h}$ ont été sacrifiés. Lors de la gestation, les mères ont reçu un régime équilibré, mais dont le taux de lipides variait. Après anesthésie rapide des animaux mâles et femelles, les tissus sont prélevés aux niveaux où des foyers de cellules adi-

\section{PLANCHE I}

FIG. 1. - Apparition des premières cellules adipeuses chez le rat, au moment de la naissance, au niveau du hile du rein. Les cellules nombreuses sont étroitement groupées autour des capillaires sanguins et forment des agrégats ( "lobules » de la littérature). 425 .

FIG. 2. - Aspect général du tissu adipeux, prélevé au niveau du hile du rein, chez un raton de $24 \mathrm{~h}$. La plupart des cellules contiennent une grosse masse de lipides entourées ou non de petites inclusions lipidiques. $\times 425$.

FIG. 3. - Coupe de fissu prélevé entre les reins chez un rat à la naissance, au stade de l'apparition des premières inclusions lipidiques. Les cellules avec des inclusions lipidiques ont un noyau, $N$, volumineux et de forme irrégulière. Les inclusions lipidiques sont très contrastées, L. Les mitochondries $M$, relativement peu nombreuses, dont les crêtes sont, dans la majorité des cas, partiellement ou complètement absentes, ont une taille et une forme variable. Nucléole, NU. $\times 3600$.

FIG. $3^{\prime}$. - Vue partielle de deux cellules voisines, du même tissu, dont les mitochondries $M$, sont très différentes par la taille, le nombre de leurs crêtes ef le contrasie de leur matrice mitochondriale. $\times 9300$. 
PI. I
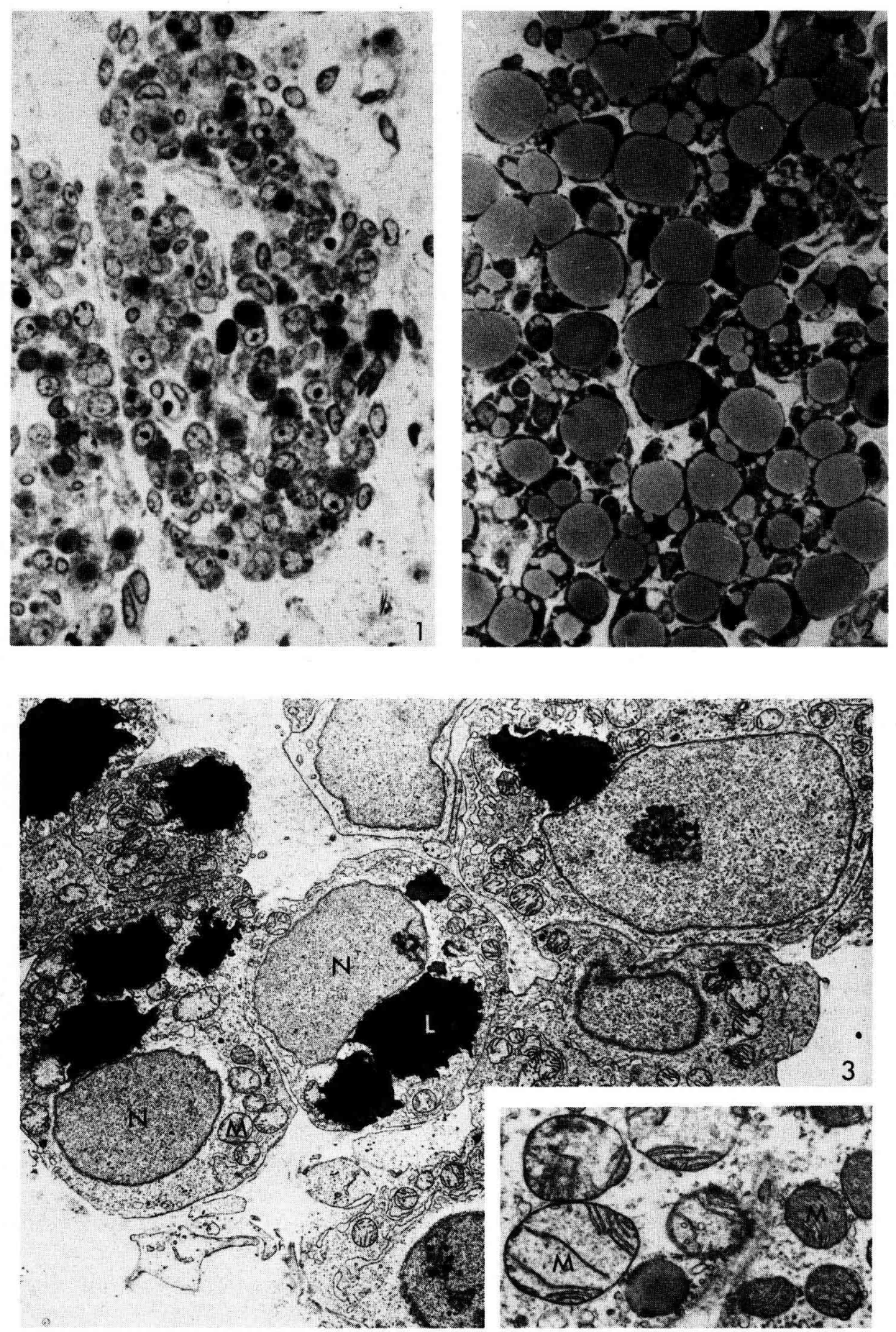
peuses sont observés soit directement, soił après immersion de la région rénale entière dans le tétroxyde d'osmium.

La fixation est faite à $4{ }^{\circ} \mathrm{C}$ dans le tétroxyde d'osmium à 2 p. 100 dans le tampon phosphate $0,1 \mathrm{M}$ selon Millonig (1961) pendant un temps variant de $2 \mathrm{~h}$ à $24 \mathrm{~h}$. Les fixations prolongées ont été faites dans le but d'obtenir une meilleure préservation des lipides (Vodovar, Desnoyers ef Flanzy, 1972 ; Desnoyers, 1973).

Après plusieurs lavages dans le tampon phosphate, les tissus sont déshydratés dans des bains d'acétone de titre croissant. L'inclusion est faite dans une « mixture » d'Epon 812 selon Luft (1961).

Les coupes semi-fines ef les coupes fines ont été préparées à partir des mêmes blocs respectivement sur le Pyramitome et sur l'Ultratome LKB. Après avoir été colorées sur lame selon la méthode de Leeson et Leeson (1970), les coupes semifines sont observées au microscope Leitz Orthoplan. Les coupes fines sont contrastées sur grille par l'acétate d'uranyl, puis par le citrate de plomb selon Reynolds (1963) et sont observées au Siemens Elmiscop $1 \mathrm{~A}$.

\section{Observations.}

1. Moment d'apparition ef localisation dans la région rénale des premières cellules avec des foyers de lipogenèse.

Sur les coupes des tissus prélevés aux différents emplacements des tissus adipeux de la région rénale, les premières cellules avec des foyers de lipogenèse ont été observées chez un nombre très réduit de fœus sacrifiés $12 \mathrm{~h}$ avant la date présumée de la naissance, chez la majorité des ratons au moment de la naissance ef chez tous les ratons âgés de $12 \mathrm{~h}$. Pour l'ensemble des ratons étudiés, les premières cellules avec des foyers de lipogenèse apparaissent simultanément en quatre localisations précises de la région rénale qui sont : le hile du rein, le «facia périrénal» ou capsule rénale, le bord interne de la loge rénale, et entre les deux reins.

\section{Observations en microscopie photonique.}

Les coupes semi-fines des tissus prélevés aux niveaux de la région rénale cités précédemment et dans lesquels apparaissent les premières cellules adipeuses, ont un aspect comparable en microscopie photonique. A tous les niveaux, les cellules sans lipides et les cellules avec des inclusions lipidiques en nombre variable sont groupées en lobules richement vascularisés et innervés et généralement limités à leur pourlour par une trame de tissu conjonctif.

Chaque lobule est formé de petites unifés de cellules disposées autour des capillaires sanguins. Cependant, comme l'ensemble des cellules d'un lobule est très resserré, l'individualité de ces petites unités n'est pas toujours nettement apparente (fig. 1). Les cellules adipeuses de ces lobules, dont la taille est fonction des lipides accumulés, ont un diamètre inférieur à $18 \mu \mathrm{m}$, quel que soit le niveau du prélèvement.

Sur les coupes de tissus prélevés $48 \mathrm{~h}$ après la naissance, les images en microscopie photonique restent comparables pour les tissus des différents niveaux ; cepen- 
dant, du fait du développement très rapide des tissus adipeux chez cette espèce, leur aspect est notablement différent de celvi observé à la naissance (fig. 2).

Au cours des premières $48 \mathrm{~h}$, l'accroissement du volume lobulaire est important, en raison de l'augmentation du volume des cellules, due aux lipides accumulés et dans une certaine proportion à l'accroissement de la population cellulaire surtout à la périphérie des lobules où les cellules sans lipides sont assez nombreuses.

Pour un nombre important de cellules, la totalité des lipides accumulés a fusionné en une seule masse et l'ensemble des éléments cellulaires non dégradés est repoussé à la périphérie de la cellule. Le diamètre de ces cellules « univacuolaires » est généralement compris entre 30 et $40 \mu \mathrm{m}$. Les autres cellules adipeuses contiennent un nombre variable d'inclusions lipidiques plus ou moins volumineuses et ont un diamètre inférieur à $30 \mu \mathrm{m}$. Enfin, les cellules sans lipide, présumées être les futurs adipocytes, sont à des stades d'évolution différents et, de ce fait, ont un aspect variable.

\section{Observations en microscopie électronique.}

Les observations en microscopie électronique ont été faites dès l'apparition des premiers foyers de lipogenèse car, chez le rat, l'accumulation des lipides dans les premières cellules adipeuses périrénales est très rapide ce qui modifie très vite leur aspect ultrastructural.

Groupées en agrégats avec les autres composants des tissus adipeux en formation, les cellules ont des formes diverses qui semblent dépendre de leur degré d'évolution, de la quantité de lipides qu'elles ont accumulés et enfin des pressions plus ou moins importantes exercées par les cellules avec lesquelles elles sont en contact.

La lame basale, qui est une des caractéristiques des cellules adipeuses, est, dans la majorité des cas, régulière et uniforme. On observe, cependant, occasionnellement que la lame basale de certaines cellules est élargie sur une portion plus ou moins importante de son pourtour ou qu'elle a fusionné avec la lame basale de la cellule avec laquelle elle se trouve en contact. Dans un nombre de cas très réduit, on constate que deux cellules adipeuses en contact ont, entre elles, une jonction assez courte de type desmosome très peu élaboré.

L'a membrane plasmique, qui suit la lame basale, est généralement régulière et bien apparente. Pour la majorité des cellules adipeuses, la membrane plasmique est pourvue d'invaginations pinocytotiques habituellement remplies par une substance dont le contraste est comparable au contraste de la lame basale.

Cependant, à part ces structures communes à toutes les cellules adipeuses périrénales, on constate que l'aspect ultrastructural des cellules adipeuses est souvent différent suivant le niveau de prélevement des tissus dans la région rénale et parfois pour des cellules se trouvant côte à côte à un même niveau.

- Les premières cellules adipeuses des tissus prélevés entre les reins (siège chez le rat, du tissu adipeux brun) ont un noyau très volumineux et de forme irrégulière aussi bien quand il occupe le centre de la cellule au début de la lipogenèse que par la suite lorsqu'il est repoussé par les lipides vers la périphérie de la cellule (fig. 3).

Les mitochondries de ces cellules sont, quelle que soit l'incidence des coupes, en partie de forme sphérique et en partie de forme plus ou moins ovale ef elles ont, en majorité, une matrice peu contrastée. Certains de ces organites ont des crêtes nom- 
breuses, pour d'autres elles sont rares et parfois inexistantes. Par ailleurs, on observe assez souvent que des cellules placées côte à côte ont, les unes des mitochondries très volumineuses avec peu de crêtes et une matrice faiblement contrastée et les autres des mitochondries de taille moyenne avec des crêtes nombreuses et une matrice bien contrastée (fig. 3). Le nombre des mitochondries, au stade de l'apparition de la lipogenèse, paraît être variable d'une cellule à l'autre, ainsi que suivant les cellules appartenant aux différents lobules. Il est faible, dans l'ensemble, par rapport à celui des cellules adipeuses des autres emplacements de la région rénale à un même stade d'évolution.

- Les cellules adipeuses des tissus prélevés au niveau des loges rénales ont un noyau de taille moyenne, de forme ovale et qui occupe une position centrale lors de l'apparition de la lipogenèse dans la cellule (fig. 4). Repoussé par les lipides au fur et à mesure de leur accumulation, il devient plus ou moins sphérique et enfin enserré entre les lipides ef la membrane plasmique il prend des formes diverses dont la plus fréquente est celle en croissant.

A ce niveau, les mitochondries ont un aspect comparable dans toutes les cellules (fig. 4). Elles sont de taille moyenne ; lorsqu'elles sont coupées suivant leur grand axe elles ont en majorité une forme de bâtonnet, mais des formes sphériques ou ramifiées sont également observées. Leurs crêles sont généralement nombreuses et assez régulières, leur matrice est assez fortement contrastée. Du fait de leur nombre très élevé au moment de l'apparition des premières inclusions lipidiques, leur volume représente jusqu'à 40 p. 100 du volume cellulaire.

Au niveau du hile (fig. 5) et de la capsule rénale, les cellules ont en majorité un noyau comparable à celui des cellules des loges rénales, tandis qu'un faible pourcentage de cellules a un noyau volumineux ef de forme irrégulière, semblable à celui des cellules des tissus prélevés entre les reins.

Les mitochondries des cellules de ces deux emplacements sont, suivant les cellules ou suivant de petits groupes de cellules qui paraissent être au même degré d'évolution, de forme et de taille variées. L'aspect d'une partie des mitochondries des cellules de ces deux emplacements est comparable à celui des mitochondries observées au niveau

\section{PLANCHE II}

FIG. 4. - Coupe de tissu prélevé dans la loge rénale chez un raf à la naissance au stade de l'apparition des premières inclusions lipidiques. Les cellules et leur noyau, $N$, sont de forme assez régulière. Les inclusions lipidiques, $L$, ont une forme plus régulière que dans la figure 3 . Les très nombreuses mitochondries, $M$, généralement en forme de bâtonnet, sont de taille variable et ont des crêtes régulières ef une matrice mitochondriale uniformément contrastée. Lame basale B; capillaire sanguin, $C$; membrane plasmique, MP. $\times 3600$.

FIG. 5. - Aspect du tissu adipeux, au stade de formation, prélevé au niveau du hile du rein, chez un rat à la naissance. Les cellules sans ef avec des inclusions lipidiques, L, sont étroitement groupées autour des capillaires sanguins, $C$. Les mitochondries, $M$, sont moins abondantes et de forme moins régulière qu'au niveau de la loge rénale. Noyau, $N$; nucléole, NU. $\times 3600$. 

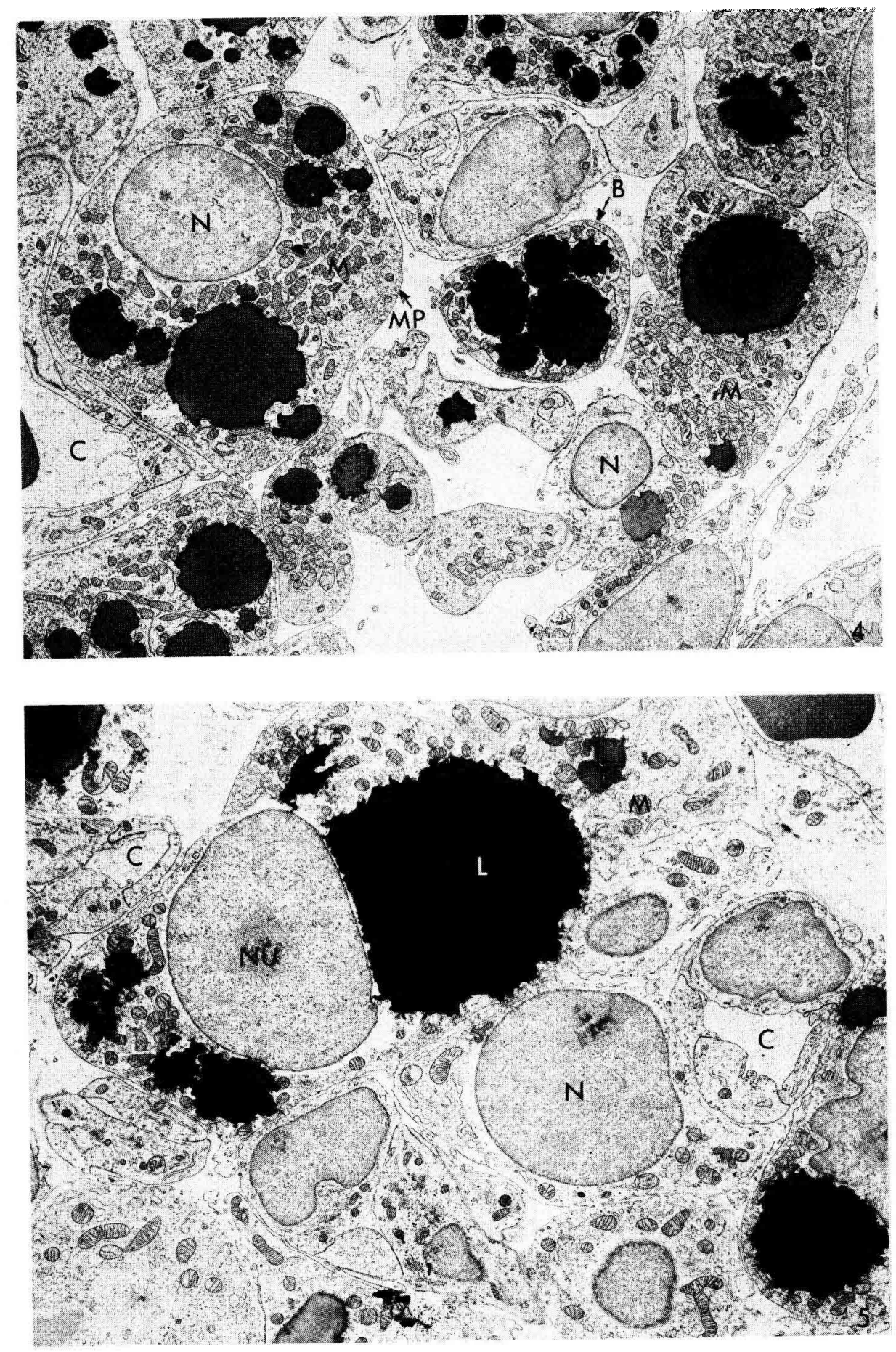
des cellules des loges rénales, l'autre partie pouvant être comparée à celui observé pour les cellules des tissus prélevés entre les deux reins.

Pour l'ensemble des emplacements étudiés dans la région rénale, l'appareil de Golgi, plus ou moins volumineux selon les cellules, estlocalisé dans la région périnucléaire ou plus rarement dans les extensions cytoplasmiques. Dès que la quantité de lipides accumulés dans une cellule devient importante, l'appareil de Golgi n'est plus observé et seuls quelques fragments d'éléments Golgiens sont encore présents entre les lipides et la membrane plasmique.

Le reticulum endoplasmique rugueux (RER) des cellules au début de la lipogenèse est relativement abondant dans les extensions cytoplasmiques et presque inexistant dans la région périnucléaire. Du fait de cette distribution et suivant l'incidence des coupes, on peut penser que les cellules sont soit riches, soit pauvres en RER. A ce stade du développement, la lumière réticulaire est souvent élargie et contient une substance plus ou moins contrastée. Au cours du remplissage des cellules pàr les lipides, le RER se fragmente et devient plus rare, tandis que l'on note une augmentation du reticulum endosplasmique lisse (REL) principalement sous forme de courts fragments et de vésicules de diamètre variabble.

En ce qui concerne les inclusions lipidiques, on constate que les cellules qui ont leurs premiers foyers de lipogenèse au moment de la naissance, sont $24 \mathrm{~h}$ ou au plus $48 \mathrm{~h}$ plus tard, pratiquement remplies de lipides. A ce moment, dans un nombre important de cellules, les inclusions lipidiques multiples ont fusionné en une masse lipidique unique ce qui permet de dire que les cellules adipeuses périrénales atteignent rapidement le stade adipocyte, mais ce qui ne veut pas dire que les cellules sont parvenues au maximum de leur volume.

A la naissance et immédiatement après, les inclusions lipidiques des cellules périrénales sont très contrastées, leur pourtour est peu régulier dans les cellules siłuées entre les reins et se rapproche de la forme sphérique au niveau de la loge rénale.

\section{Origine des cellules adipeuses périrénales de rat.}

Au cours de cette étude, les observations concernant l'origine des cellules adipeuses se sont révélées complexes du fait de la diversité de la population cellulaire à l'emplacement de ces tissus avant et pendant l'apparition des premiers foyers de lipogenèse. Néanmoins, nous avons pu noter une abondante formation de capillaires sanguins sur les coupes des tissus prélevés aux niveaux du hile rénal, de la capsule rénale et de la loge rénale, lorsque l'on s'approche du moment d'apparition de la lipogenèse. Au moment du bourgeonnement des capillaires sanguins, des cellules endothéliales apparaissent en nombre variable. Tout d'abord, les cellules endothéliales qui font partie du bourgeon capillaire sont attachées aux parois vasculaires par la lame basale des capillaires, puis ces cellules entourées de leur propre lame basale se détachent de la paroi des capillaires et restent disséminées autour de ceux-ci. Enfin, aux alentours de la naissance, les foyers de lipogenèse apparaissent dans ces cellules. Ces observations nous autorisent donc à penser que la grande majorité, sinon la totalité, des cellules adipeuses périrénales des tissus éfudiés aux emplacements indiqués ci-dessus, sont d'origine endothéliale comme les cellules adipeuses internes à graisse blanche des autres emplacements précédemment étudiés. 
Pour ce qui est des cellules adipeuses apparaissant entre les reins (cellules du futur tissu adipeux brun) et dont l'aspect ultrastructural comme nous l'avons montré est assez particulier, nos observations actuelles ne nous permettent pas de conclure quant à leur provenance.

\section{Discussion.}

L'investigation histocytologique du tissu adipeux périrénal du rat, au stade périnatal, nous a permis de mettre en évidence chez cette espèce, le moment d'apparition des premières cellules avec des inclusions lipidiques et de localiser leur distribution dans la région rénale.

Nous avons pu constater que, pour le rat, l'âge auquel les premiers foyers de cellules adipeuses apparaissent est le même pour l'ensemble des animaux sacrifiés de celte espèce.

Cela corrobore les observations que nous avons faites chez d'autres espèces pour le même tissu adipeux (Desnoyers, Vodovar, 1977) ou pour d'autres tissus adipeux internes (Vodovar, Desnoyers et François, 1971 ; Desnoyers et Vodovar, 1974) et permet de penser que l'apparition de la lipogénèse aux différents emplacements est liée à l'espèce. Ainsi, chez le rat ef d'une façon constante, tous les tissus adipeux apparaissent aux alentours de la naissance, c'est-à-dire que 2 à 5 jours seulement séparent les premières cellules adipeuses périrénales, qui apparaissent en premier lieu, des premières cellules adipeuses mésentériques qui opparaissent les dernières. Chez le porc, comme nous l'avons montré précédemment (Desnoyers et Vodovar, 1977), le tissu adipeux périrénal apparaît très précocement au stade fœtal chez le fotus âgé d'environ 70 jours comme les tissus adipeux épididymaire et péricardiaque (Desnoyers ef Vodovar, 1974) ; seul fait exception, le tissu adipeux mésentérique qui apparaît chez le fœetus âgé d'environ 104 jours (Vodovar, Desnoyers ef François, 1971).

La distribution dans la région rénale des premiers foyers de cellules adipeuses à partir desquels s'édifient les tissus adipeux périrénaux du rat, est comparable à celle observée chez le fœus de porc (Desnoyers et Vodovar, 1977) et semble être prédéterminée comme le sont, en général, les emplacements des tissus adipeux dans l'organisme de chaque espèce (Vodovar, Desnoyers et François, 1971 ; Desnoyers, 1973).

Les modalités de cette prédétermination des premières cellules adipeuses dans l'organisme n'ont pas encore été élucidées. Cependant, à la suite de nos observations, il nous semble que les formations cellulaires décrites par certains auteurs (Wassermann, 1926) comme des « organes primitifs» précurseurs des tissus adipeux sont probablement le stade premier de la prolifération cellulaire au moment du bourgeonnement des capillaires sanguins.

Nos observations concernant l'origine et le mode d'apparition des cellules adipeuses périrénales autour des capillaires sanguins, puis la formation des agrégats cellulaires, sont comparables à ce qui a été décrit chez le porc pour lestissus adipeux mésentériques (Vodovar, Desnoyers et François, 1971) et pour les tissus adipeux péricardiaque et épididymaire (Desnoyers et Vodovar, 1974). Néanmoins, on note que d'une façon générale, les agrégats cellulaires que l'on appelle lobules, sont bien 
séparés les uns des autres chez le rat ce qui n'est pas le cas chez le porc. Cette différence s'explique aisément par la richesse relative en tissu conjonctif de ces emplacements chez le rat.

En comparant les cellules adipeuses périrénales de rat au stade de l'apparition de la lipogenèse à celle du porc au même stade, on constate que les organites cellulaires sont beaúcoup plus abondants chez la première espèce ce qui laisse supposer une activité cellulaire plus importante. Cela se traduit par un remplissage des cellules adipeuses par les lipides beaucoup plus rapide chez le rat que chez le porc.

Par ailleurs, on note qu'il existe chez le rat., au moment de l'apparition des inclusions lipidiques, un nombre important de préadipocytes à un stade avancé d'évolution, contrairement à ce que nous avons observé chez le fotus de porc (Desnoyers et Vodovar 1977) chez qui les cellules se remplissent au fur et à mesure de leur prolifération.

Le fait que nous ayons constaté qu'au stade périnatal les cellules adipeuses de rat, présumées être à un même degré d'évolution, ont un aspect ultrastructural distinct suivant le niveau étudié de la région rénale, en particulier au niveau de la loge rénale et entre les deux reins, peut s'expliquer par une différence de nature des cellules adipeuses de ces deux emplacements. En effet, chez le rat, on trouve entre les reins du tissu adipeux brun (Afzelius, 1970), alors qu'au niveau de la loge rénale il s'agit de tissu adipeux blanc.

En ce qui concerne le polymorphisme de la population mitochondriale des cellules adipeuses brunes, nos observations confirment les travoux antérieurs faits au niveau des tissus adipeux interscapulaires de la même espèce (Napolitano et Fawcett, 1958). Néanmoins, nous avons noté que les premières cellules adipeuses qui apparaissent entre les reins ne sont comparables ni aux cellules des tissus adipeux bruns interscapulaires du même rat, ni aux cellules du tissus adipeux au même niveau chez un rat de 7 jours.

Au premier abord, on peut penser que ces différences pourraient être attribuées au fait que les cellules sont à des stades d'évolution différents, étant donné que le tissu adipeux interscapulaire apparaît plus précocement que le tissu adipeux situé entre les reins.

Cependant, si on considère l'aspect général des premières cellules apparaissant entre les reins et la quantité importante de lipides fusionnés en une seule masse qu'elles contiennent rapidement, on conçoit difficilement qu'en peu de temps ces cellules puissent prendre l'aspect, classiquement décrit des cellules des tissus adipeux bruns (Napolitano et Fawcett, 1958 ; Picard, Tasso et Cotte, 1966 ; Suter, 1969). Une hypothèse serait que ces premières cellules sont rapidement renouvelées et qu'elles sont remplacées par des cellules caractéristiques des tissus adipeux bruns.

Enfin, pour ce qui est des tissus adipeux du hile du rein et de la capsule rénale, dont le développement ultérieur est toujours très limité, il semble qu'il puisse y avoir deux possibilités : soit que les cellules adipeuses à graisse brune se transforment en cellules adipeuses à graisse blanche, soit qu'il y ait, au stade primaire, une étroite interpénétration des deux types de cellules et que, par la suite, seules les cellules adipeuses à graisse blanche prolifèrent.

Réunion Groupe Développement INRA/Productions animales Thiverval-Grignon, 14-15 avril 1976. 
Remerciements. - Cette étude a été réalisée avec l'appui financier de I'INSERM (ATP no 19) et de I'INRA (ATP «Tissu adipeux»).

\section{Références}

AFZELIUS B. A., 1970. Brown adipose tissue : its gross anatomy, histology and cytology, 1-31. In LINDBERG O., Brown adipose tissue, Elsevier, N.-Y.

DESNOYERS F., 1973. Etude morphologique des adipocytes et leur évolution chez le porc et chez le rat. Thèse Doct., Paris.

DESNOYERS F., VODOVAR N., 1973. La lame basale de la cellule adipeuse à graisse blanche et son évolution au cours de l'amaigrissement. C. R. Acad. Sci. Paris, D, 276, 2285-2287.

DESNOYERS F., VODOVAR N., DURAND G., 1973. Transformation ef devenir des cellules adipeuses au cours de l'amaigrissement. Ełude ultrastructurale. Ann. Biol. anim. Bioch. Biophys., 13, 75-92.

DESNOYERS F., VODOVAR N., 1974. Apparition, origine et évolution des tissus adipeux épididymaire et péricardiaque du fœtus de porc. Ann. Biol. anim. Bioch. Biophys., 14, 769-780.

DESNOYERS F., VODOVAR N., DURAND G., 1975. Observations ultrastructurales, chez le rat, de cellules adipeuses totalement dépourvues de lipides d'accumulation, puis de leur remplissage de novo. C. R. Acad. Sci. Paris D, 280, 1717-1720.

DESNOYERS F., VODOVAR N., 1977. Etude structurale et ultrastructurale du tissu adipeux périrénal au siade de sa formation chez le porc. Ann. Biol. anim. Bioch. Biophys., 17, 269-282.

HERMANS P. G. C., 1973. The development of adipose tissue in swine fœuses (a morphological study). Tijdschr. Diergeneesk., 98, 662-667.

LEESON C. R., LEESON T. S., 1970. Staining methods for sections of epon embedded tissues for light microscopy. Can. J. Zool., 48, 189-190.

LEMONNIER D., 1972. Effect of âge, sexe and site on the cellularity of the adipose tissue in mice and rat rendered obese by a high fat dief. J. clin. Invest., 51, 2907-2915.

LUFT J., 1961. Improvments in epoxy resin embedding methods. J. biophys. biochem. Cytol., 9, 409-414.

MILLONIG G., 1961. The advantages of a phosphate buffer for OsO4 solutions in fixation. J. appl. Physics, 32, 1637.

NAPOLITANO L., 1963. The differenciation of white adipose cells. J. Cell Biol., 18, 663-679.

NAPOLITANO L., 1965. Observations on the fine structure of adipose cells. Ann. N. Y. Acad. Sci., 131, 34-42.

NAPOLITANO L., FAWCETT D., 1958. The fine structure of brown adipose tissue in the newborn mouse and rat. J. biophys. biochem. Cytol., 4, 685-703.

NOUGUÈS J., 1975. Adipocyłe growth of four adipose deposits in rabbit. Ann. Biol. anim. Bioch. Biophys., 15, 541-546.

PICARD D., TASSO F., COTTE G., 1966. Aspect ultrastructural de la lipogenèse dans le tissu adipeux brun. Z. Zellforsch., 69, 260-273.

REYNOLDS E., 1963. The use of lead citrate at high $\mathrm{pH}$ as an electron opaque stain in electron microscopy. J. Cell Biol., 17, 208-212.

SHELDON H., 1965. Morphology of adipose tissue : an microscopic anatomy of fat. In REYNOLD A. E., CANILL G. F. Jr. Handbook of physiology : adipose tissue vol. 5, 125-139, Am. Phys. Soc., Washington D. C.

SHELDON H., 1969. The morphology and growth of adipose tissue. Proc. Meat Ind. Res. Conf., 9-23.

SHELDON H., HOLLENBERG C. H., WINEGRAD A. I., 1962. Observation on the morphology of adipose tissue. The fine structure of cells from fasted and diabetic rats. Diabetes, 11, 378-387.

SIMON G., 1962. Genesis and structure of adipose tissue in man. Acta anat., 48, 232-241.

SUTER E. R., 1969. The fine structure of brown adipose tissue. Labor. Invest., 21, 246-258.

VODOVAR N., SERRES F., FRANÇOIS A. C., 1969a. Aspect morphologique de la formation du dépôt adipeux mésentérique chez le porcelet. C. $R$. Acad. Sci. Paris D, 269, 969-971.

VODOVAR N., SERRES F., FRANÇOIS A. C., 1969b. Evolution ultrastructurale des cellules adipeuses du mésentère du porcelet. C. R. Acad. Sci., Paris D, 262, 919-921. 
VODOVAR N., DESNOYERS F., FRANÇOIS A. C., 1971. Origine et évolution des adipoc yte mésentériques du porcelet avant la naissance. Aspect ultrastructural. J. Microsc., 11, 265-284.

VODOVAR N., DESNOYERS F., FLANZY J., 1972. Dépôt mésentérique du porcelet : étude morphologique. Ann. Biol. anim. Bioch. Biophys., 12, 243-262.

WASSERMANN F., 1926. Die Fettorgane des Menschen. Entwicklung, Bau und systematische Stellung des sogenannten Fettgewebes. Z. Zellforsch. Mikr. Anat., 3, 235.

WASSERMANN F., 1965. The development of adipose tissue. In REYNOLD A. E., CAHILL G. F. Jr. Handbook of physiology : adipose tissue, 5, 87-100, Am. Phys., Soc., Washington D. C.

WASSERMANN F., McDONALD T. F., 1963. Electron microscopic study of adipose tissue (fat organs) with special reference to the transport of lipids between blood and fat cells. Z. Zellforsch., 59, 326-357. 